\title{
Erratum to: Statistical consideration of propeller load fluctuation at racing condition in irregular waves
}

Oleksiy Bondarenko • Masashi Kashiwagi

Published online: 29 November 2011

(C) JASNAOE 2011

Erratum to: J Mar Sci Technol

DOI 10.1007/s00773-011-0136-z

The article cited above was incorrectly categorized. The correct category is Original Article, not Review Article.

The online version of the original article can be found under doi:10.1007/s00773-011-0136-z.

O. Bondarenko $(\bowtie) \cdot$ M. Kashiwagi

Graduate School of Engineering, Osaka University,

2-1 Yamada-oka, Suita, Osaka 565-0871, Japan

e-mail: Oleksiy_Bondarenko@naoe.eng.osaka-u.ac.jp 